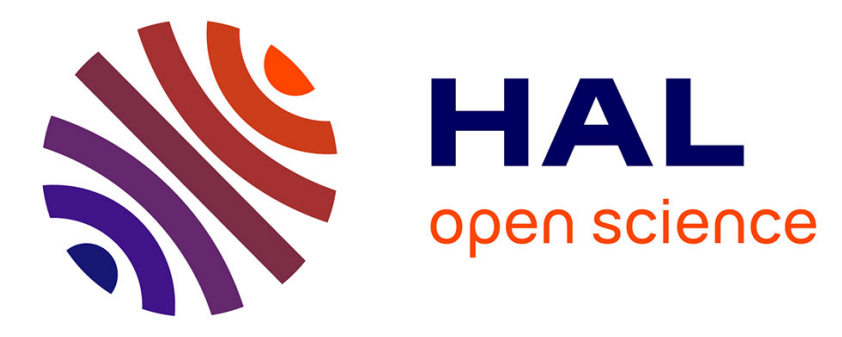

\title{
Geometrical variations management for additive manufactured product
}

Jean-Yves Dantan, Zhicheng Huang, Edoh Goka, Lazhar Homri, Alain

Etienne, Nicolas Bonnet, Mickael Rivette

\section{- To cite this version:}

Jean-Yves Dantan, Zhicheng Huang, Edoh Goka, Lazhar Homri, Alain Etienne, et al.. Geometrical variations management for additive manufactured product. CIRP Annals - Manufacturing Technology, 2017, 66 (1), pp.161-164. 10.1016/j.cirp.2017.04.034 . hal-02308242

HAL Id: hal-02308242

https://hal.science/hal-02308242

Submitted on 8 Oct 2019

HAL is a multi-disciplinary open access archive for the deposit and dissemination of scientific research documents, whether they are published or not. The documents may come from teaching and research institutions in France or abroad, or from public or private research centers.
L'archive ouverte pluridisciplinaire HAL, est destinée au dépôt et à la diffusion de documents scientifiques de niveau recherche, publiés ou non, émanant des établissements d'enseignement et de recherche français ou étrangers, des laboratoires publics ou privés. 


\title{
Geometrical variations management for additive manufactured product
}

\author{
Jean-Yves Dantan (2)*, Zhicheng Huang, Edoh Goka, Lazhar Homri, \\ Alain Etienne, Nicolas Bonnet, Mickael Rivette \\ LCFC, Arts et Métiers - ParisTech - Metz, HESAM, ENIM, 4 rue Augustin Fresnel, 57078 Metz Cedex 3, France \\ Submitted by Jean-Yves Dantan
}

\begin{abstract}
A B S T R A C T
Additive manufacturing (AM) became an advanced research topic due to its ability to manufacture complex shapes. But the ability to achieve predictable and repeatable shapes is critical. Therefore, to optimize the design of an additive manufactured product, tolerancing is a key issue. This paper focuses on geometrical quality assessment of an AM product. It includes a process oriented geometrical model to predict the surface roughness and dimensional deviations, and a geometrical simulation tool to assess the impacts of these deviations on the geometrical behaviour of the joint. An application of the approach is illustrated through a case study.
\end{abstract}

\section{Introduction}

The development of new advanced additive manufacturing techniques has progressed greatly in the recent years. These new additive manufacturing (AM) techniques affect the manufacturing strategy of new products. They accelerate innovation, reduce the cost of supply chains, and reduce the waste [1].

To improve these new AM techniques and to increase the scope of their applications, research activities require to overcome some key technical challenges, mainly the following regards as critical: to achieve predictable and repeatable shapes. Process variability must be reduced, as must the sensitivity to process variations (the impact of these variations on the assembly behaviour).

To investigate the dimensional and geometrical accuracy (process variability) for new AM techniques, various designs of test artefact have been developed [2]. Standard test artefacts incorporate multiple features. The combination of these features provides a global assessment of the Geometrical Dimensioning and Tolerancing (GD\&T) characteristics: "a cube is used for perpendicularity, parallelism, linear accuracy and surface finish evaluation and a cylindrical hole is used to evaluate the roundness, cylindricity, radius accuracy and positioning accuracy. Some test artefacts incorporate some specific AM features: overhanging features, freeform geometries, features for part warpage and stairs" [2]. Most test artefact applications are based on traditional GD\&T characteristics, and they provide a global assessment proposed in the International Tolerance Grade of the AM techniques defined in ISO 286 [2]. In fact, the aim of these applications is the identification of influential process factors on the geometrical accuracy of additive manufactured parts; usually, this identification is based on Design of Experiments [3].
To investigate the dimensional and geometrical accuracy, another approach is the identification of predictive model of the additive manufactured parts geometrical defects [4].

As mentioned in the second paragraph, there exist two solutions to improve the geometrical quality of an additive manufactured product: the mitigation of the process variability and the mitigation of the sensitivity of the process variations. This paper focuses on the second solution and its tolerancing. To mitigate these variabilities, it is firstly required to model them and to identify what are the process and product design drivers involved. Being able to model them can help designers to assess, before the manufacturing of the part and/or product, what will probably be final shapes of the manufactured product to verify if functional surfaces remain in an acceptable range.

This paper is divided into two main sections: Section 1 presents the skin model representations of AM part, the mathematical models for the geometrical behaviour prediction of an AM assembly and an approach for AM tolerance analysis are illustrated in Section 2.

\section{Surface roughness and dimensional deviations prediction of AM part}

Tolerance analysis aims to simulate the "real-product" with the minimum uncertainty. This uncertainty is partly due to the geometrical deviation representations. A significant amount of research efforts has been carried out in the last decade to explore the mathematical models for geometric deviation representation: variational geometry approach, skin model shape, modal representation, etc.

The variational geometry approaches [5] are based on the parameterization of deviations from theoretic geometry. The real geometry of parts is considered by a variation of nominal dimension or it is apprehended by a variation (position and 


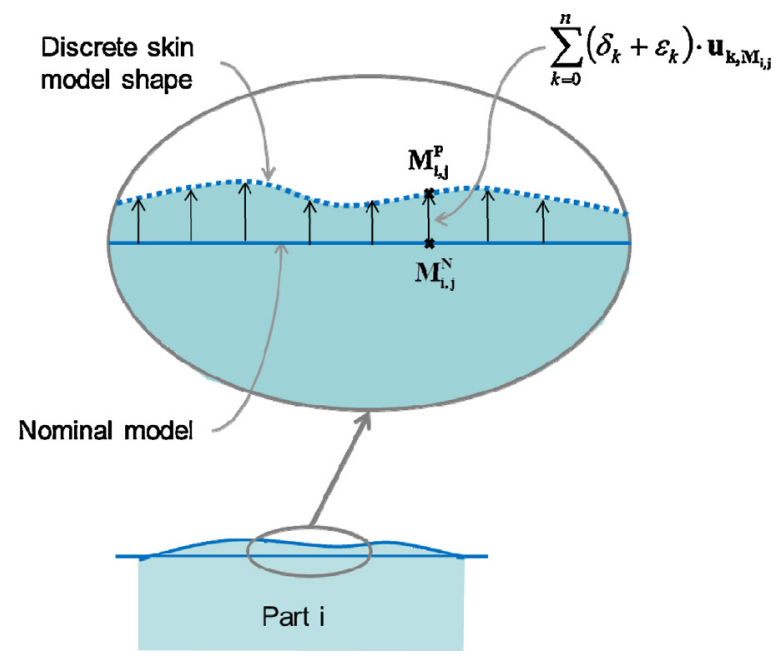

Fig. 1. Discrete skin model shape illustration and description.

orientation) of the nominal geometry. In this approach, the form defects are neglected. The representation of the skin model has been investigated only recently. Anwer et al. [6] proposed a comprehensive framework for skin model simulation. This representation includes position, orientation and form defects. The modal representation method of geometrical deviation decomposition has extensively been studied. Huang et al. [7] proposed discretecosine-transformation (DCT) based on decomposition method for form defects modelling. Samper et al. [8] developed the Discrete Modal Decomposition (DMD) considering modal shapes of a discretized feature. Usually, the technical interpretation of these modal representations is not easily achieved.

Based on these concepts, a skin model representation is proposed to predict the geometrical quality of additive manufactured parts. This representation is simplified to obtain a finite description like a discrete shape. To define the discrete shape model, the nominal shape is sampled into a set of points, and the discrete skin model shape is apprehended by the displacement of each point, leading to a large number of parameters (Fig. 1). To reduce the number of parameters, a geometrical deviation decomposition based on the definition of process oriented geometrical defect modes is proposed:

Substitute surface model $=$ No minal surface model

+ Position defect mode + Orientation defect mode

+ Form defect mode due to the mesh

+ Form defect mode due to layers strategy

+ Form defect mode due to the geometrical defects

of the machine

$+\ldots$

The amplitude of each deviation includes a systematic component $\delta_{k}$ and an aleatory component $\varepsilon_{k}$. Therefore, the skin model representation (Fig. 1) of each surface in the local coordinate system is given by:

$\mathbf{O}_{\mathbf{i}} \mathbf{M}_{\mathbf{i}, \mathbf{j}}^{\mathbf{P}}=\mathbf{O}_{\mathbf{i}} \mathbf{M}_{\mathbf{i}, \mathbf{j}}^{\mathbf{N}}+\sum_{k=0}^{n}\left(\delta_{k}+\epsilon_{k}\right) \cdot \mathbf{u}_{\mathbf{k}, \mathbf{M}} \mathbf{M}_{\mathbf{i} \mathbf{j}}$

with $\mathbf{Q}_{\mathbf{i}}$ : datum of the feature i, $\mathbf{O}_{\mathbf{i}} \mathbf{M}_{\mathbf{i}, \mathbf{j}}^{\mathbf{P}}$ : coordinates of the predicted point $j$ of the feature $i, \mathbf{O}_{\mathbf{i}} \mathbf{M}_{\mathbf{i}, \mathbf{j}}^{\mathbf{N}}$ : coordinates of the nominal point $j$ of the feature $\mathrm{i}, \mathbf{u}_{\mathbf{k}, \mathbf{M}_{\mathbf{i}, \mathrm{j}}}$ : kth modal deviation expressed at the point $\mathrm{M}_{\mathrm{i}, \mathrm{j}}$, $\delta_{k}$ : systematic component of the amplitude of the kth mode, $\epsilon_{k}$ : aleatory component of the amplitude of the kth mode.

Based on the additive manufacturing processes knowledge, this modal representation is established. The amplitude assessment is the result of a set of experiments and fitting operations between the measured points and the modal representation. The built defect modes can be thus predicted for an AM geometrical feature with satisfactory accuracy.
To better illustrate the developed model, a simplified test artefact is proposed as a case study and Fused Deposition Modeling (FDM) is studied. This test artefact incorporates three cylinders with radiuses from $10 \mathrm{~mm}, 20 \mathrm{~mm}$, to $30 \mathrm{~mm}$ respectively in order to identify the relationship between defect mode caused deviations and the part design parameters. All test artefacts were printed on the Replicator $2 \times$ FDM printer using $1.75 \mathrm{~mm}$ diameter PLA filament and they were measured using an coordinate measuring machine (CMM) with an accuracy of less than $4 \mu \mathrm{m}$ (around 5000 measured points for each cylinder).

Geometrical defect modes (Fig. 2) for AM manufactured cylinders are modelled and generated by considering additive manufacturing process characteristics. When transform the CAD model to STL file, the mesh generation operation would turn the section of cylinder into polygon which is defined as the Mesh Mode in this study. While manufacturing parts, FDM extrudes heated material deposited on a substrate though a movable nozzle and cooled down until it solidifies before new layer is deposited. The "Layer upon layer" method as well as the deposited material shape would cause the Layer Mode. The shrinkage after cooling down would result in the Radius Mode, etc. Machine movement control defect would form the Ellipse Mode and Rounded Rectangle Mode. Geometrical deviation of the machine is also considered in this study, defect mode caused by the gap in the machine moving axis is defined as the Gap Mode. This modal representation could include others technical modes.

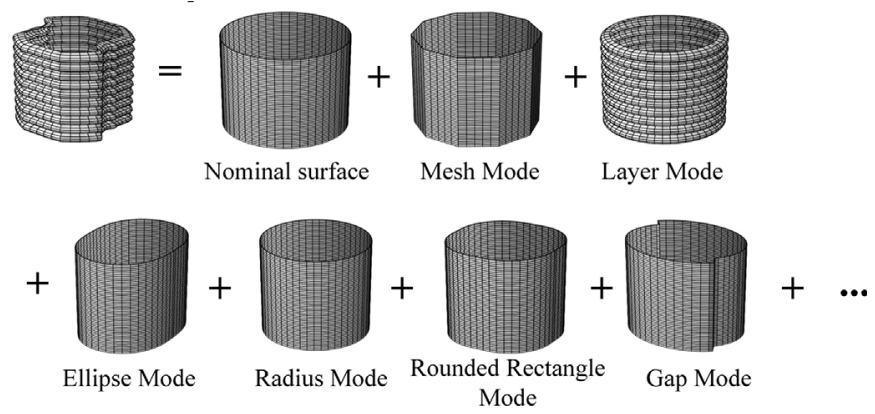

Fig. 2. Cylindrical prediction surface generation with process oriented geometrical defect modes.

The final cylindrical prediction surface shape is resulted by the combination of the defect modes added onto the nominal surface as Fig. 2 shows.

The identification of the systematic and aleatory components of the modal representation is performed on the measured points of test parts by the iterative least square method. To highlight the impact of the AM form defects, Table 1 contains a comparison between the results of a fitting operation of cylinders (without form defect modelling) and the results of the identification of the

Table 1

Prediction result and defect modes caused deviations (mm).

\begin{tabular}{|c|c|c|c|}
\hline \multirow[b]{2}{*}{ Measurement accuracy } & Radius 10 & Radius 20 & Radius 30 \\
\hline & \multicolumn{3}{|l|}{0.004} \\
\hline \multicolumn{4}{|c|}{ Distance between measured points and cylinders } \\
\hline Max distance & 0.183 & 0.244 & 0.306 \\
\hline Average distance & 0.107 & 0.156 & 0.232 \\
\hline Standard deviation & 0.053 & 0.042 & 0.049 \\
\hline \multicolumn{4}{|c|}{$\begin{array}{l}\text { Distance between measured points and prediction points after applying the } \\
\text { geometrical defect modes }\end{array}$} \\
\hline Max distance & 0.072 & 0.088 & 0.075 \\
\hline Average distance & 0.018 & 0.015 & 0.019 \\
\hline Standard deviation & 0.013 & 0.011 & 0.014 \\
\hline \multicolumn{4}{|c|}{ Deviation caused by each geometrical defect mode } \\
\hline Mesh Mode & 0.007 & 0.013 & 0.003 \\
\hline Layer Mode & 0.019 & 0.006 & 0.005 \\
\hline Radius Mode & 0.208 & 0.273 & 0.358 \\
\hline Ellipse Mode & 0.116 & 0.081 & 0.073 \\
\hline Rounded Rectangle Mode & 0.025 & 0.035 & 0.044 \\
\hline Gap Mode & 0.051 & 0.049 & 0.042 \\
\hline
\end{tabular}


predicted model: the accuracy of the predicted model is ten times greater than that of the cylinder (maximum value of the distances and average of the distances). The averages and the standard deviations characterize the aleatory components of each model. And the last part of Table 1 contains the values of all systematic components of the case study.

As shown in the table, form defects of AM parts cannot be ignored when assembly simulation. The proposed model integrates the two components of the manufacturing imprecisions: systematic and aleatory.

\section{Geometrical behaviour prediction of the AM assembly}

Tolerance analysis includes three main issues [5,9]: geometrical deviations modelling, system behaviour with deviations modelling and tolerance analysis developing. The first issue for AM was detailed in the previous section. The following section focuses on the two last issues.

\subsection{Geometrical simulation with deviations}

In order to build the geometrical behaviour model, it is necessary to define the surface deviations $X=\left\{\delta_{1}, \ldots, \delta_{n}, \varepsilon_{1}, \ldots, \varepsilon_{n}\right\}$ of each component (Section 2) and the relative displacements between nominal components according to the gap $G=\left\{g_{1}, \ldots, g_{m}\right\}$ (Fig. 3).

The geometrical simulation of assemblies has been carried out in several existing works [9]. The behaviour modelling of AM assembly means the definition a mathematical model to characterize the system behaviour with deviations. Several approaches to handle with geometrical constraints when modelling admissible displacements between parts in assembly have been proposed. Giordano et al. [10] introduce the concept of deviations and clearance domains. The model has been extended by Zou et al. [11] and Homri et al. [12]. Most of these approaches did not take parts form defects into account.

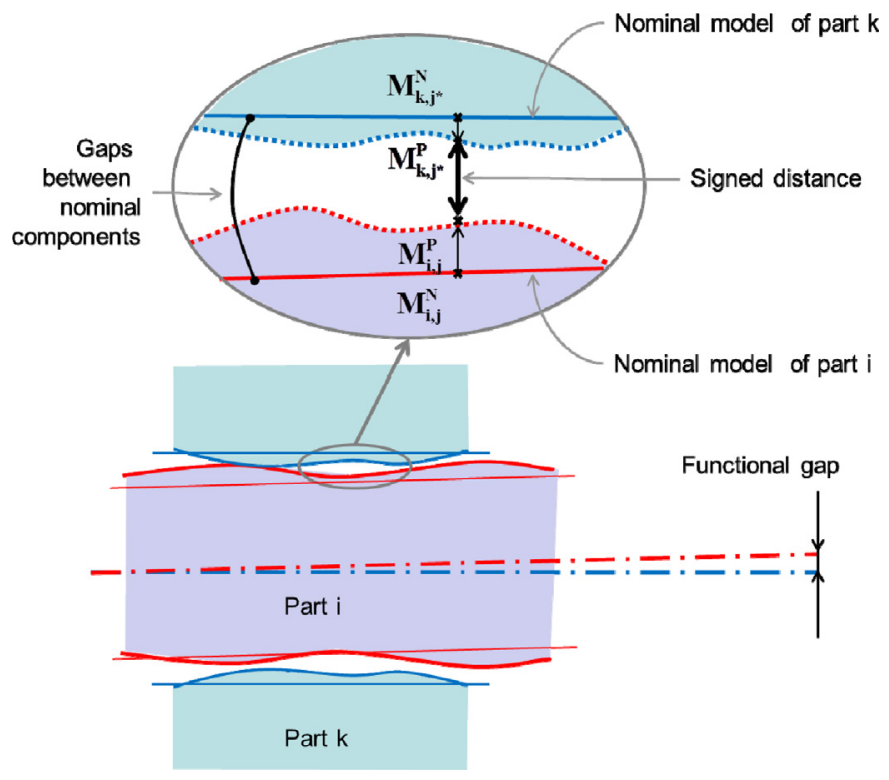

Fig. 3. Joint model.

As shown in Section 2, the form defects of AM parts cannot be ignored. These should be considered in the computation of the assembly simulation of AM parts. Anwer et al. [6] define the relative positioning of non-ideal parts as the relative positioning of the two associated skin model shapes by optimization. Dantan [13] compares three techniques of contact analysis which take into account the geometrical deviations. The discrete shape representation and the simplified contact analysis approach offer the best compromise between the accuracy and the computing time.
Based on these approaches, the proposed mathematical formulation of tolerance analysis is built on the expression of the geometrical behaviour of the assembly:

- Compatibility equations (As datum chains, composition relations of displacements in the various topological loops define compatibility equations between parts deviations and gaps) [14].

- Interface constraints (interface constraints limit the geometrical behaviour of the assembly and characterize non-interference or association between skin model shapes, which are nominally in contact) [14].

The interface constraints are affected by the form defects. Therefore, it is necessary to traduce the non-interference between the two surfaces: The signed distance $e\left(M_{i, j}^{P}\right)$ between two discrete skin model shapes which are nominally in contact should be positive or zero (Fig. 3 and Eq. (3)). This signed distance depends on the surface deviations and gaps [14].

$\forall j \quad e\left(M_{i, j}^{P}\right) \geq 0$

with

$e\left(M_{i, j}^{P}\right)=\mathbf{M}_{\mathbf{i} \mathbf{j}}^{\mathbf{P}} \mathbf{M}_{\mathbf{k} \mathbf{j} *}^{\mathbf{P} *} \cdot \mathbf{n}_{\mathbf{i} \mathbf{j}}$

$\left\|\mathbf{M}_{\mathbf{i}, \mathbf{j}}^{\mathbf{P}} \mathbf{M}_{\mathbf{k} \mathbf{j} *}^{\mathbf{P}} \wedge \mathbf{n}_{\mathbf{i}, \mathbf{j}}\right\|=0$

where $\mathbf{n}_{\mathbf{i}, \mathbf{j}}$ is the normal vector at the point $\mathbf{M}_{\mathbf{i}, \mathbf{j}}^{\mathbf{N}}$.

These constraints allows to the worst admissible configurations of the assembly.

\subsection{Analysis method}

The goal of tolerance analysis is to predict a quality during the design stage. Therefore, the main objective of the analysis method is to estimate:

- the probability of the assemblability,

- the probability of respect of the functional requirements, for a given tolerance specification.

The probability of the assemblability is equal to the probability of the existence of gaps that ensure the assembly of the components including part deviations, that is also equal to the probability of the existence of an admissible gap configuration of the assembly such that the interface constraints and the compatibility equations are respected.

In a similar way, the probability of respect of the functional requirements is equal to the probability that, for all admissible gap configurations of the assembly, the interface constraints, the compatibility equations and the functional requirement are satisfied.

The proposed method for the probability estimation combines a Monte Carlo simulation and an optimization algorithm. The existences of an admissible gap configuration or the worst gap configuration identification are performed by the minimization of one function such that all compatibility and interface constraints are satisfied. The optimization problem can be defined by Eq. (4) [14].

Minimize $_{\text {Gaps }} f(\ldots)$

Subject to $\forall(i, j) \quad e\left(M_{i, j}^{P}\right) \geq 0, \ldots$

The number of considered points of the discrete skin model shape has a great impact on the estimated probability accuracy as well as the computing time. To analyze the computing time with relation to the number of considered points of the discrete skin model shape, several algorithms were tested [14]. A linear quadratic programming solver (one of the best algorithms [14]) is applied on the test case and the results are shown in Fig. 4. The computing time increases exponentially. 


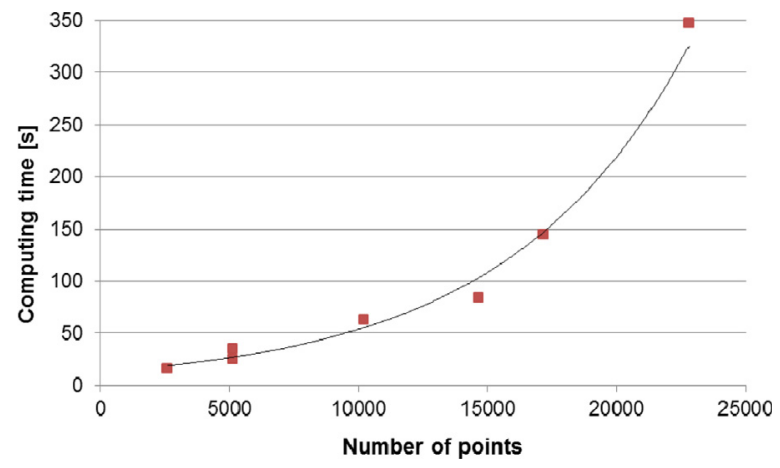

Fig. 4. Computing time in relation to the number of points.

Moreover, to illustrate the significant impact of the AM form defects, some results of three simulations of a considered functional gap of cylindrical joint (see Fig. 3) are given in Table 2. Form defects are not firstly considered in the simulation; the simulation 2 and 3 are two Monte Carlo simulations with AM form defects defined respectively in an important point density and a low point density.

Table 2

Prediction results of the worst configuration of the considered functional gap ( $\mathrm{mm}$ ).

\begin{tabular}{llll}
\hline & \multicolumn{3}{l}{ Simulation } \\
\cline { 2 - 4 } & 1 & 2 & 3 \\
\hline Worst value of the worst configurations & 0.405 & 0.742 & 0.638 \\
Mean value of the worst configurations & & 0.363 & 0.361 \\
Standard deviation of the worst configurations & & 0.213 & 0.145 \\
\hline
\end{tabular}

These modelling and techniques are tested on an assembly (Fig. 5). Without form defect, the probability of the assemblability is equal to $100 \%$; with form defect, this probability is equal to $82 \%$. These results were computed by Monte Carlo simulation $(10,000$ iterations) coupled by optimization (linear quadratic programming solver). These algorithms were coded in SCILAB ${ }^{\circledR}$ environment and run using a computer with Intel Xeon ${ }^{\mathbb{R}}$ CPU ES 1607. The computing time increases with the density. In addition, it is not possible to know a priori for which density value the probability of the assemblability has converged. In this case, the upper bound of the computing time is $22 \mathrm{~h}$.

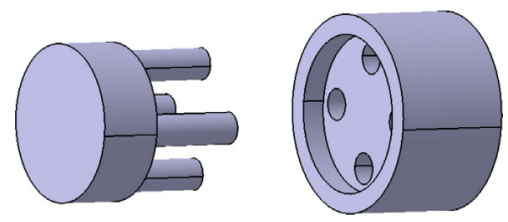

Fig. 5. Assembly.

\section{Conclusion}

During the design stage, the new paradigm of additive manufacturing requires to manage the manufacturing imperfections. The assessment of the quality level of an assembly is a key element which enables to improve the functional quality of the assembly. To assess this quality level, some mathematical models are required, which must represent the manufacturing imprecisions and the assembly behaviour as well as possible.

Investigations of dimensional accuracy by various AM processes have received important attention in the literature. Dimensional tolerances rankings are developed for additive manufacturing. Most of these investigations focus on the linear dimension.

To improve the geometrical variations management of additive manufactured product, it is necessary to increase the accuracy of the AM geometrical defect characterization. In fact, the dimensional tolerance rankings are not sufficient to simulate the impact of most AM defects on the product behaviour. To do so, the discrete skin model shape combined with the definition of a modal representation of AM geometrical defects was performed. This proposed modal representation is based on the Additive Manufacturing signature. The results point out that the form defects need to be considered as well. The proposed modal representation includes some systematic components and some aleatory components which aim to modelize the "real world".

As the AM form defect cannot be neglected, one technique of geometrical simulation with deviations was proposed and tested. The results confirm the importance of the AM form defect.

To improve the AM product quality during the design phase, the proposed approach allows an optimal characterization of the AM geometrical deviations and the analysis of design of the AM product on the quality.

\section{References}

[1] Thompson MK, Moroni G, Vaneker T, Fadel G, Campbell RI, Gibson I, Bernard A Schulz J, Graf P, Ahuja B, Martina F (2016) Design for Additive Manufacturing: Trends, Opportunities, Considerations, and Constraints. Annals of the CIRP 65(2):737-760.

[2] Lieneke T, Adam G, Leuders S, Knoop F, Josupeit S, Delfs P, Zimmer D (2015) Systematical Determination of Tolerances for Additive Manufacturing by Measuring Linear Dimensions. 26th Annual International Solid Freeform Fabrication Symposium, Austin, Texas, USA, 371-384.

[3] Shahrain M, Didier T, Kheng Lim G, Qureshi AJ (2016) Fast Deviation Simulation for 'Fused Deposition Modeling' Process. Procedia CIRP 43:327-332.

[4] Huang Q, Nouri H, Xu K, Chen Y, Sosina S, Dasgupta T (2014) Predictive Modeling of Geometric Deviations of 3D Printed Products - A Unified Modeling Approach for Cylindrical and Polygon Shapes. IEEE International Conference on Automation Science and Engineering (CASE), Taipei, 25-30.

[5] Dantan JY, Gayton N, Dumas N, Etienne A, Qureshi AJ (2012) Mathematical issues in Mechanical Tolerance Analysis. AIP PRIMECA 2012, France.

[6] Anwer N, Schleich B, Mathieu L, Wartzack S (2014) From Solid Modelling to Skin Model Shapes: Shifting Paradigms in Computer-Aided Tolerancing. Annals of the CIRP 63(1):137-140.

[7] Huang W, Ceglarek D (2002) Mode-based Decomposition of Part Form Error by Discrete-Cosine-Transform with Implementation to Assembly and Stamping System with Compliant Parts. Annals of the CIRP 51(1):21-26.

[8] Grandjean J, Ledoux Y, Samper S, Favrelière H (2013) Form Errors Impact in a Rotating Plane Surface Assembly. Procedia CIRP 10:178-185.

[9] Dantan JY (2014) Tolerancing, CIRP Encyclopedia of Production Engineering, Springer: $1230-1237$.

[10] Giordano M, Duret D (1993) Clearance Space and Deviation Space: Application to Three-Dimensional Chain of Dimensions and Positions. 3rd CIRP Design Seminar on Computer-Aided Tolerancing, France.

[11] Zou Z, Morse E (2004) A Gap-Based Approach to Capture Fitting Conditions for Mechanical Assembly. Computer-Aided Design 36(8):691-700.

[12] Homri L, Teissandier D, Ballu A (2015) Tolerance Analysis by Polytopes: Taking into Account Degrees of Freedom With Cap Half-Spaces. Computer-Aided Design 62:112-130.

[13] Dantan JY (2015) Comparison of Skin Model Representations and Tooth Contact Analysis Techniques for Gear Tolerance Analysis. ASME Journal of Computing and Information Science in Engineering 15(2).

[14] Homri L, Dantan JY, Levasseur G (2016) Comparison of Optimization Techniques in a Tolerance Analysis Approach Considering Form Defects. Procedia CIRP 43:184-189. 